\title{
27. ON THE RADIANT DISTRIBUTION OF SPORADIC METEORS
}

\author{
N. Carrara, A. Consortini, and L. Ronchi \\ (Centro Microonde of Consiglio Nazionale delle Ricerche, Florence, Italy)
}

\begin{abstract}
A research is described which had the purpose of deriving information about the physics and astronomy of sporadic meteors from the range distribution of radar echoes from meteor trails experimentally observed under given conditions.

The method is based on the comparison of the experimental distribution with a number of different theoretical distributions, corresponding to different sets of assumptions about the characteristics of sporadic meteors and the law of trail formation.

The quantities which can be derived by this method are: (1) the distribution of heliocentric radiants, (2) the type of meteors (stone, iron, dustballs), (3) the distribution of heliocentric velocities.

In the present paper are described the results of the first part of the theoretical computations. Evidence is obtained that, in order to fit the experimental curve, both the uniform distribution of heliocentric radiants over the celestial sphere and in the plane of the ecliptic must be taken into account.
\end{abstract}

The purpose of the present paper is to describe research carried out at the Centro Microonde to derive information about the physics and astronomy of sporadic meteors from the range distribution of radar echoes from meteor trails experimentally observed under given conditions.

The experimentally observed range distribution (O.R.D.) used in our analysis is shown in Figure 1. The data for it were collected (Burlamacchi et al., 1965) in the periods August 10 to August 13, 1962 and 1963, with the radar set for meteor observations in use at the Centro Microonde from 1959 (Carrara, 1966). The antenna was pointed towards North and the observations were carried out from 05.00 to $06 \cdot 30$. The total number of echoes is $\sim 1500$.

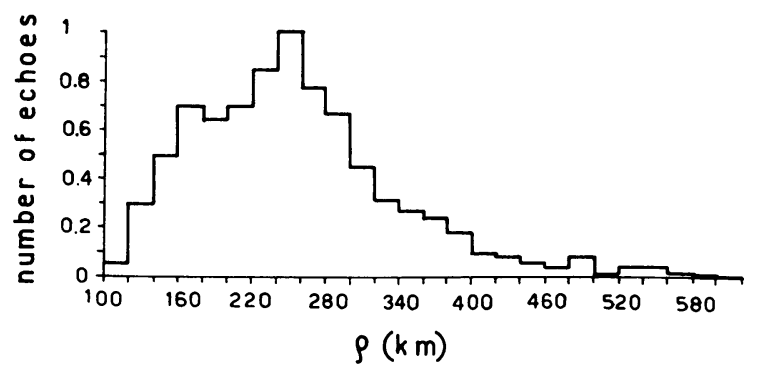

FIG. 1. The experimentally observed range distribution. 
The method of analysis is based on the comparison of the histogram of Figure 1 with a number of different theoretical histograms (T.R.D.), each one corresponding to a different set of assumptions about the characteristics of sporadic meteor flow and the law of trail formation. The analysis is not yet completed; however, the preliminary results we are now presenting, already seem to provide significant information.

The theoretical background is described in a number of papers and reports (see, e.g., Kaiser, 1954a, b, 1960, 1961 ; Carrara et al., 1960a, b, 1962, 1965). Here we only recall that its application implies the knowledge of a number of quantities, namely:

(a) the characteristics of the apparatus (radiation pattern, gain and bearing of the antenna, radiated wavelength, total radiated power, threshold power),

(b) atmospheric quantities (pressure, gravity acceleration and scale-height as functions of altitude),

(c) quantities involved in the process of trail formation (latent heat of evaporation, mean atomic weight of meteors, ionization probability and its dependence on the geocentric velocity of meteors).

The features of the apparatus were determined as accurately as possible, as described by Burlamacchi et al. (1965) and Carrara (1966).

The atmospheric model was deduced from a previous analysis of the range distribution due to the meteors of Perseid shower. The experimental data used in such an analysis were collected at the same time and with the same apparatus as those reported in Figure 1. To this end, during the experiments, the antenna had been alternatively pointed $15 \mathrm{~min}$ towards North and $15 \mathrm{~min}$ towards South. Note that for the particular geometry and time, no echo from shower meteors is observed with the North bearing of the antenna, so that Figure 1 collects only data from sporadic meteors. The histogram corresponding to the South bearing of the antenna includes also shower echoes and was used both to check the method of analysis and to find the best-fitting atmospheric model (Handbook of Geophysics, 1960) of the T.R.D. with the O.R.D., as described by Carrara et al. $(1966 a, b)$.

The quantities involved in the process of trail formation were taken from the literature (Öpik, 1958; Verniani, 1964).

In evaluating the T.R.D.'s which will now be presented, we assumed the validity of the Herlofson theory of ablation. We recall that it only serves to deduce the minimum meteor mass detectable by a given apparatus operating in given conditions, as a function of the velocity, direction and altitude of the trail as well as of the features of the apparatus. When the fragmentation theory is available, all the treatment will be applied again.

The various T.R.D.'s differ from one another as regards (1) the assumed (heliocentric) radiant distribution, (2) the type of meteors, (3) the dependence of the ionization probability on the geocentric velocity of the meteors, (4) the range of the heliocentric velocity of the meteors.

As to the radiant distribution, we consider two possibilities, namely, (1a) radiants 
uniformly distributed over the celestial sphere, (1b) radiants uniformly distributed in the plane of the ecliptic.

As to the meteor type, we considered three cases: (2a) stone meteors, (2b) iron meteors, (2c) dustballs. In the first case the mass density $\delta=3.4 \mathrm{gr} \mathrm{cm}^{-3}$ and the mean atomic weight $\mu=23$; in the second case $\delta=7 \cdot 8 \mathrm{gr} \mathrm{cm}^{-3}, \mu=56$; in the third case $\delta=0.2 \mathrm{gr} \mathrm{cm}^{-3}, \mu=23$. The last value of $\delta$, which is found in the literature of some years ago (Verniani, 1964) is now replaced by a slightly higher value (Verniani and Hawkins, 1965), but we took it as a trial value.

As to the dependence of the ionization probability $\gamma$ on the geocentric velocity $v$, we considered two cases (Kaiser, 1953; Verniani and Hawkins, 1964): (3a) $\gamma=\gamma_{0}$ with $\gamma_{0}=0 \cdot 1$, and (3b) $\gamma=\gamma_{0}^{\prime} v^{4}$ with $\gamma_{0}^{\prime}=10^{-8}$ and $v$ measured in $\mathrm{km} / \mathrm{sec}$.

Finally, as regards the intervals of heliocentric velocities $V$, we considered the intervals $0-10 \mathrm{~km} / \mathrm{sec}, 10-20 \mathrm{~km} / \mathrm{sec}, 20-30 \mathrm{~km} / \mathrm{sec}, 30-40 \mathrm{~km} / \mathrm{sec}$.

The T.R.D.'s obtained under assumption (1a) have recently been published by Carrara et al. (1967). They are reported here in Figures 2 and 3. A common feature is that the T.R.D.'s are only slightly dependent on the heliocentric velocity.

Figure 4 refers to assumption ( $1 \mathrm{~b})$ and $(3 \mathrm{~b})$, that is to meteors with heliocentric radiants uniformly distributed in the plane of the ecliptic and $\gamma=\gamma_{0}^{\prime} v^{4}$.

In the case of assumption ( $1 \mathrm{~b}$ ) it turned out that the time variations of the T.R.D.'s within the period of observation were not negligible, and had to be taken into account by a time integration. From an insight of Figure 4, it appears also that the dependence on the heliocentric velocity is not negligible.

The calculations under assumption (1b) must still be completed by taking into account assumption (3a), that is $\gamma=\gamma_{0}$.

Table 1 summarizes the main results of the calculations, reporting the ranges of the maxima of the histograms shown in Figures 2, 3, and 4. It appears that such ranges pass from 180-200 km (iron and stone meteors with radiants distributed over the celestial sphere) to $240-280 \mathrm{~km}$ (dustballs with radiants distributed over the plane of the ecliptic and heliocentric velocity in the interval $20-40 \mathrm{~km} / \mathrm{sec}$ ).

Let us now come back to Figure 1. We see that the O.R.D. presents a pronounced maximum at $240-260 \mathrm{~km}$ and a plateau at $160-220 \mathrm{~km}$. From the preceding results we may conclude that the maximum at $240-260 \mathrm{~km}$ provides an evidence of the presence of light meteors (dustballs) with radiants uniformly distributed on the ecliptic, while the low-range portion of the O.R.D. can be explained only by invoking iron or stone meteors with radiants uniformly distributed over the celestial sphere. This point is evidenced by the histograms of Figure 5, where the experimental histogram is compared with the histograms (d) of iron meteors of Figure 2 (Figure 5a), of dustballs of Figure 2 (Figure $5 \mathrm{~b}$ ), of iron meteors of Figure 4 (Figure $5 \mathrm{c}$ ) and of dustballs of Figure 4 (Figure 5d). Stony meteors have not been taken into account, but the corresponding histograms are only slightly different from those of iron meteors.

We now look for the linear combination of the different T.R.D.'s which best fits the 


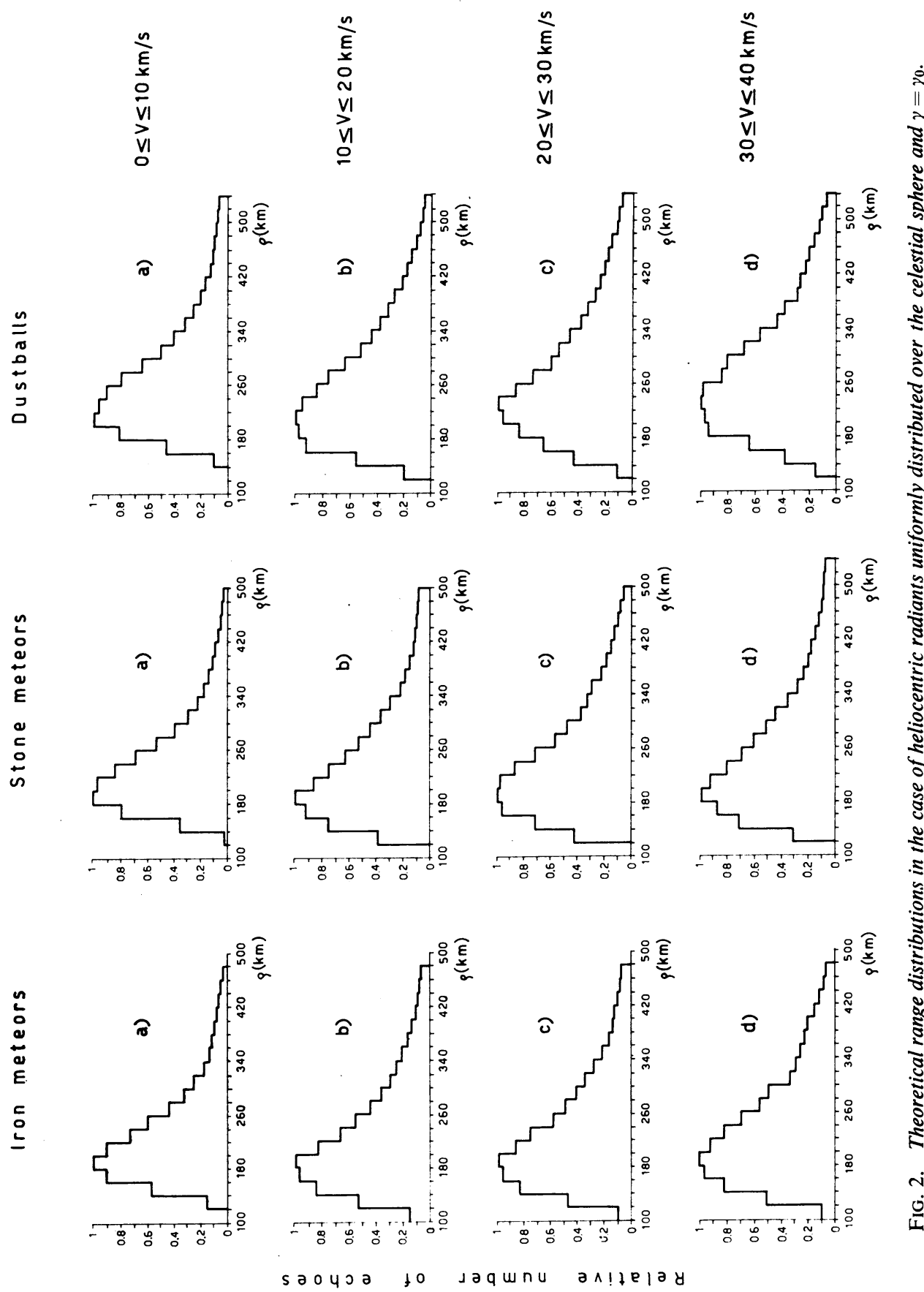



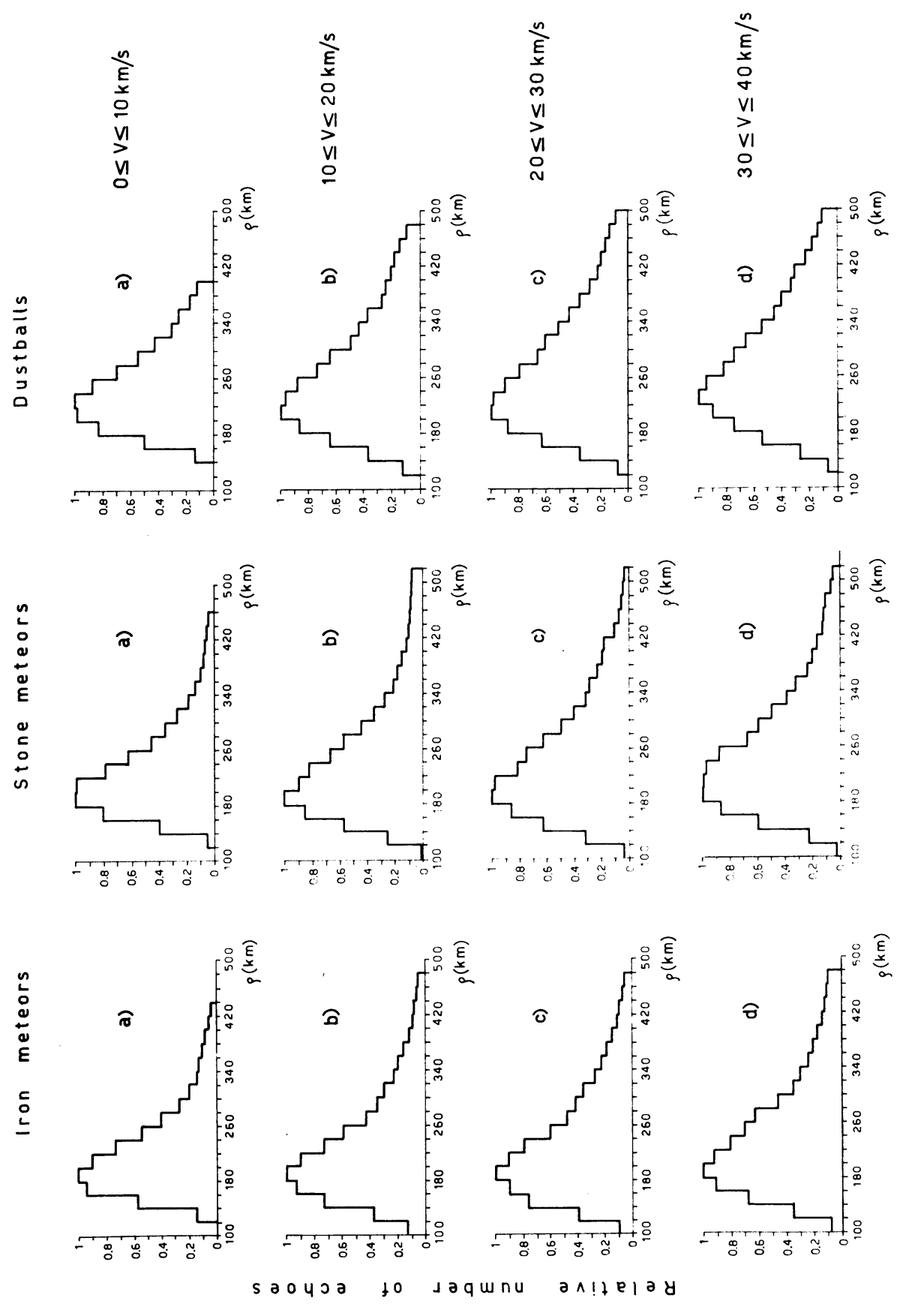

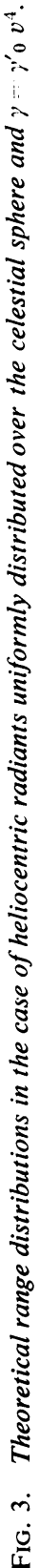



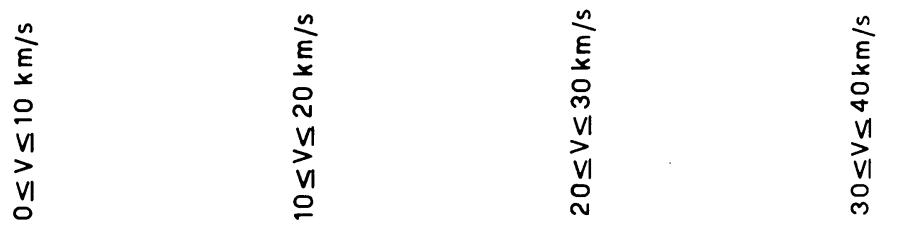

(⿸丆口
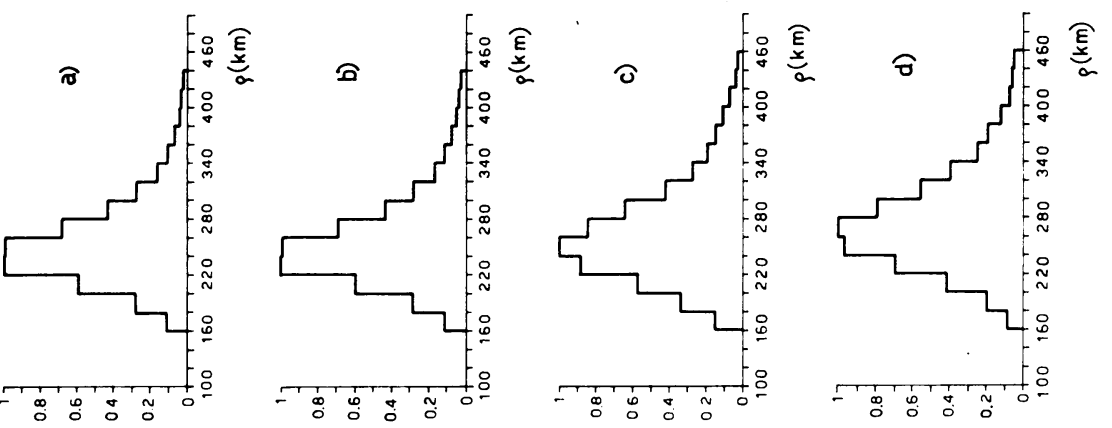

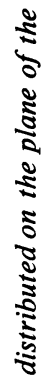
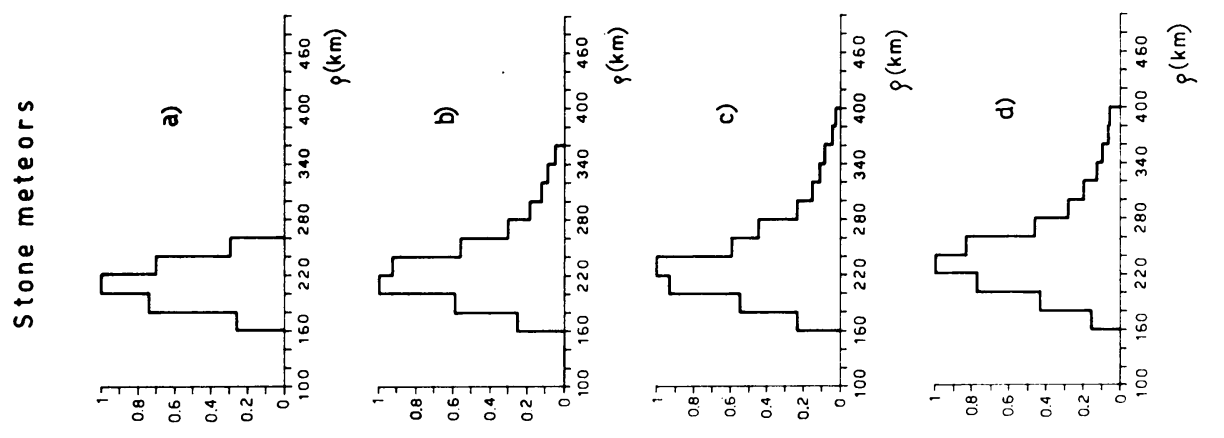

है

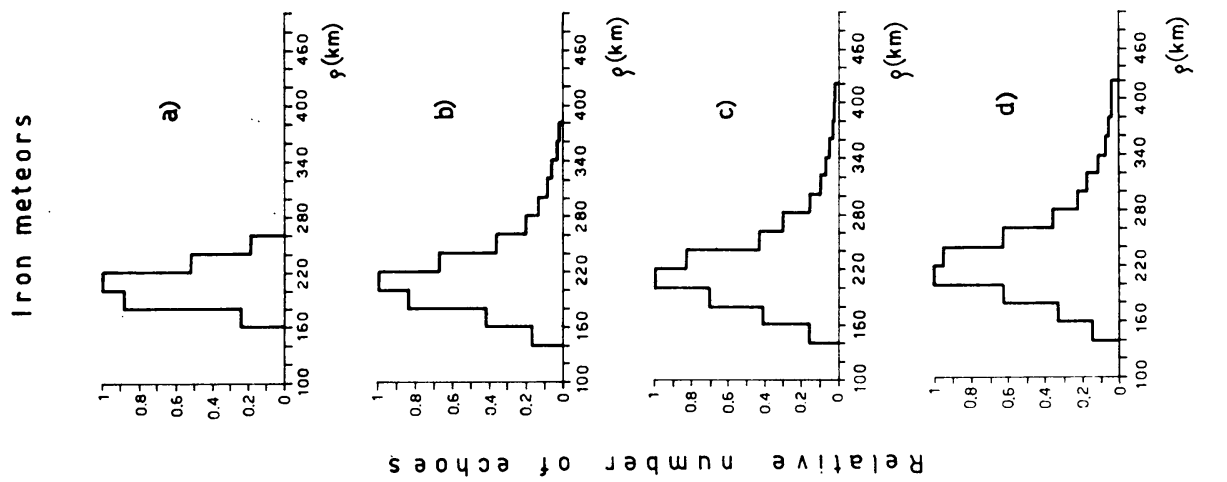




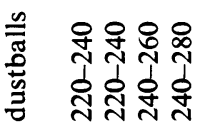

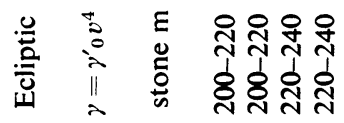

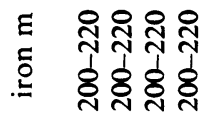

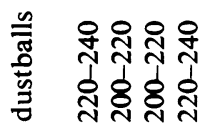

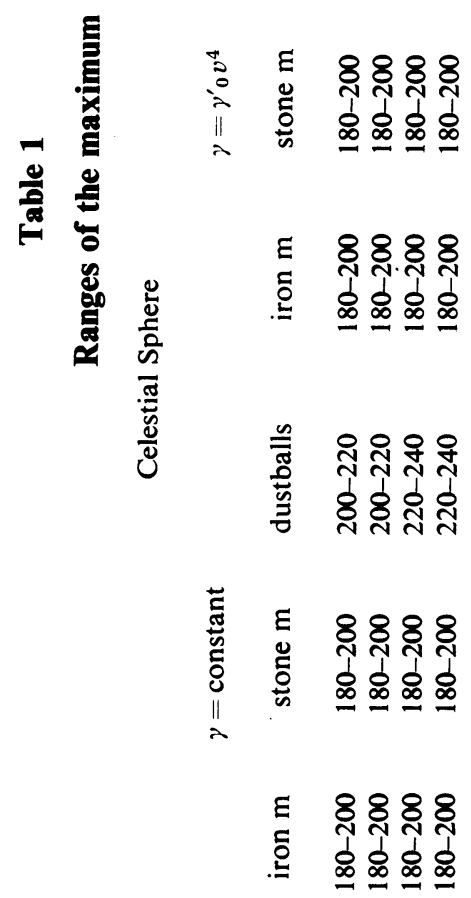

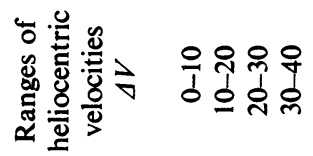



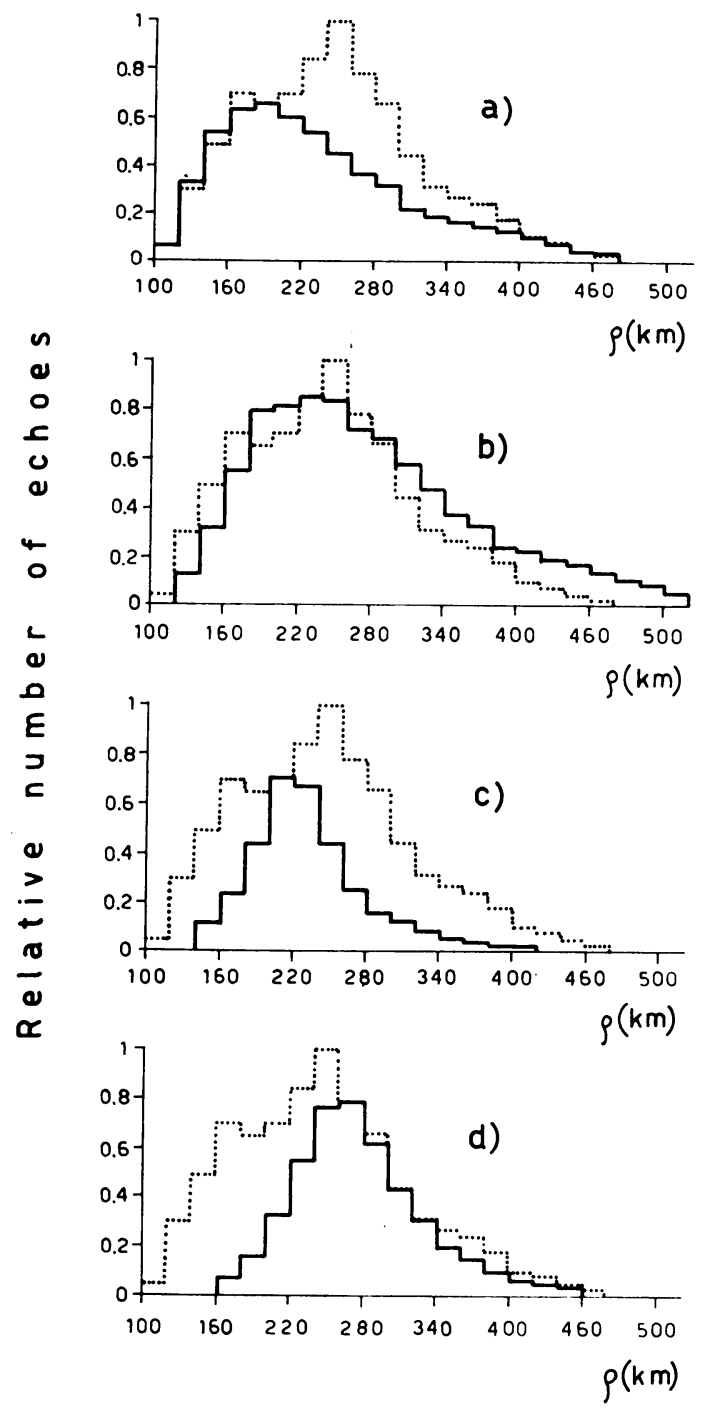

Fig. 5. Comparison of the O.R.D. (dotted line) and four T.R.D.'s (solid lines).

O.R.D. However, it must be emphasized that the linear combination must be built up only with positive coefficients. The criterion for the best fit is to minimize the meansquared error. This method will be fully applied when the calculations have been completed. For the moment we considered combinations of only two theoretical histograms, one of which is chosen between the histograms (c) and (d) of dustballs of Figure 4, and the other one is chosen among the histograms (b), (c) and (d) of iron meteors of Figure 2. 
The reason for this choice is that the selected histograms appeared at a first sight to correspond to a better fitting than the others. However, this assumption will be checked in a future analysis.

The best-fitting linear combination we obtained is shown in Figure 6 (solid line) together with the experimental histogram (dotted line), and involves the histogram (d) of iron meteors of Figure 2 (with a coefficient 0.60 ) and the histogram (d) of dustballs of Figure 4 (with a coefficient $0 \cdot 49$ ).

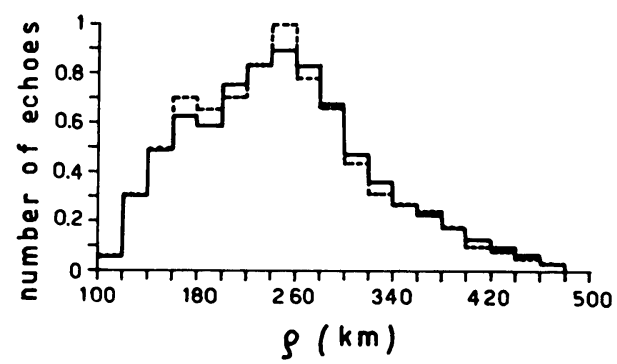

FIG. 6. The best-fitting theoretical range distribution (solid line) and the experimentally observed range distribution (dotted line).

Though these results are only preliminary, we note that, considering the presence of fluctuations in the experimental data, the agreement of the two histograms of Figure 6 looks satisfactory. When all the calculations are completed, it is expected that the agreement will be a little better.

As a conclusion, we want to emphasize that the analysis of the range distributions of the radar echoes from meteor trails seems to be a powerful means for deriving information about the physics and astronomy of the sporadic meteors.

\section{Acknowledgements}

We express our thanks to the Centro Nazionale Universitario di Calcolo Elettronico in Pisa (Italy), for having made the computations of this work with the I.B.M. 7090. Thanks are also due to Dr. M.E. De Langer for her help in carrying out the computations.

The work has been partly supported by the Ministero della Pubblica Istruzione and partly by the Consiglio Nazionale delle Ricerche.

\section{References}

Burlamacchi, P. et al. (1965) Centro Microonde Rep., No. 20, 1.5.

Carrara, N. (1966) Atti Fond. G. Ronchi, 21, 131.

Carrara, N., Checcacci, P.F., Ronchi, L. (1960a) Proc. I.R.E., 48, 2031. 
Carrara, N., Checcacci, P.F., Ronchi, L. (1962) Nuovo Cim., 24, 145.

Carrara, N., Checcacci, P. F., Consortini, A., Ronchi, L. (1960b) Alta Frequenza, 29, 615.

Carrara, N., Checcacci, P. F., Consortini, A., Ronchi, L. (1965) Nuovo Cim. Suppl., 3, 1029.

Carrara, N., Consortini, A., De Langer, M. E., Ronchi, L. (1966a) Nuovo Cim., 43B, 186.

Carrara, N., Consortini, A., De Langer, M.E., Ronchi, L. (1966b) Atti Fond. G. Ronchi, 21, 422.

Carrara, N., Consortini, A., De Langer, M.E., Ronchi, L. (1967) Centro Microonde Rep., No. $55,1.9$.

Handbook of Geophysics (1960) Macmillan Co., New York.

Kaiser, T.R. (1953) Phil. Mag. Suppl., 2, 495.

Kaiser, T. R. (1954a) Mon. Not. R. astr. Soc., 114, 39.

Kaiser, T. R. (1954b) Mon. Not. R. astr. Soc., 114, 52.

Kaiser, T.R. (1960) Mon. Not. R. astr. Soc., 121, 284.

Kaiser, T.R. (1961) Mon. Not. R. astr. Soc., 123, 265.

Öpik, E.J. (1958) Physics of Meteor Flight in the Atmosphere, Interscience Publishers, New York.

Verniani, F. (1964) Nuovo Cim., 33, 1173.

Verniani, F., Hawkins, G.S. (1964) Astrophys. J., 140, 1590.

Verniani, F., Hawkins, G.S. (1965) Harvard Radio Meteor Project Rep., No. 12.

\section{DISCUSSION}

Elford: What mass-distribution function was used in the calculation of the range-distribution curves?

Cionini: According to the available literature it was assumed that the mass distribution of sporadic meteors follows the law

$$
f(m) \mathrm{d} m=K / m^{2} \times \mathrm{d} m,
$$

where $f(m) \mathrm{d} m$ represents the probability for a meteor to have a mass between $m$ and $m+\mathrm{d} m$, and $K$ is a dimensional constant.

Lindblad: At what time of the day and year were your range-distribution data collected?

Cionini: Our range distribution data were collected from observations made in the periods August 10-13, 1962 and 1963 from 05:00 to 06:30 local time. The total number of echoes is about 1500.

Glöde: Bearing in mind that the range distribution strongly depends on the aerial diagram, how did you correct your range observations and what kind of antenna did you use?

Cionini: We know the strong dependence of the range distribution on the aerial diagram. In fact we measured it by means of an airplane equipped with a fixed frequency transmitter and flying at a constant altitude above the ground. Our antenna consisted of two identical four-element Yagi arrays, horizontally mounted at a height of $\lambda / 2$ over the ground (see Carrara, 1966).

Fedynskij: The method is interesting. But unfortunately the chosen parameter - the range distribution - is not sensitive to the investigated quantity (the density of meteor bodies).

Cionini: Thank you for your comment. I am not of your opinion. Please be so kind as to read the theoretical background of the presented method in the published paper: N. Carrara et al., A Method of Investigation of the Physics and Astronomy of Radiometeors, Suppl. Nuovo Cimento, 4, 1965 Series I, Vol. 3, pp. 1029-1063. 